\title{
Practical Guide to Single-Protein AFM Nanomechanical Spectroscopy Mapping: Insights and Pitfalls As Unraveled by All- Atom MD Simulations on Immunoglobulin G
}

\author{
J. G. Vilhena,* Maria Ortega, Manuel R. Uhlig, Ricardo Garcia, and Rubén Pérez*
}

Cite This: ACS Sens. 2021, 6, 553-564

Read Online

ACCESS | Llll Metrics \& More | 国 Article Recommendations | sl Supporting Information

ABSTRACT: Atomic force microscopy is an invaluable characterization tool in almost every biophysics laboratory. However, obtaining atomic/sub-nanometer resolution on single proteins has thus far remained elusive- a feat long achieved on hard substrates. In this regard, nanomechanical spectroscopy mapping may provide a viable approach to overcome this limitation. By complementing topography with mechanical properties measured locally, one may thus enhance spatial resolution at the single-protein level. In this work, we perform all-atom molecular dynamics simulations of the indentation process on a single immunoglobulin $\mathrm{G}(\mathrm{IgG})$ adsorbed on a graphene slab. Our simulations reveal three different stages as a function of strain: a noncontact regime-where the mechanical

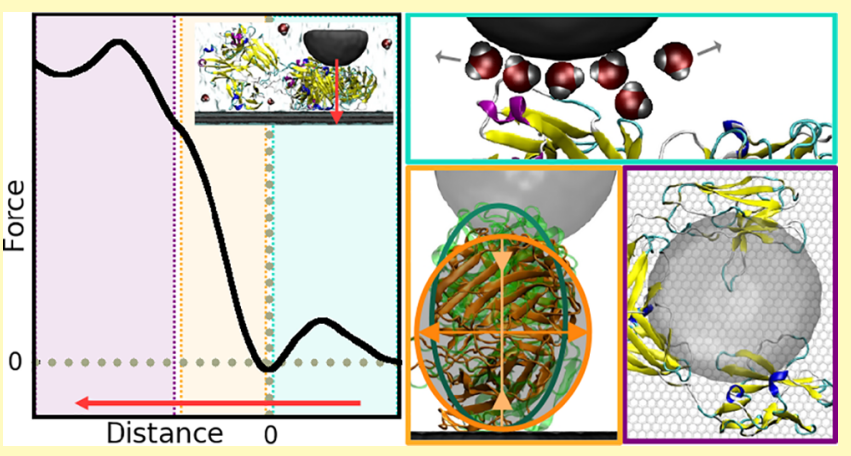
response is linked to the presence of the water environmentfollowed by an elastic response and a final plastic deformation regime. In the noncontact regime, we are able to identify hydrophobic/hydrophilic patches over the protein. This regime provides the most local mechanical information that allows one to discern different regions with similar height/topography and leads to the best spatial resolution. In the elastic regime, we conclude that the Young modulus is a well-defined property only within mechanically decoupled domains. This is caused by the fact that the elastic deformation is associated with a global reorganization of the domain. Differences in the mechanical response are large enough to clearly resolve domains within a single protein, such as the three subunits forming the IgG. Two events, unfolding or protein slipping, are observed in the plastic regime. Our simulations allow us to characterize these two processes and to provide a strategy to identify them in the force curves. Finally, we elaborate on possible challenges that could hamper the interpretation of such experiments/simulations and how to overcome them. All in all, our simulations provide a detailed picture of nanomechanical spectroscopy mapping on single proteins, showing its potential and the challenges that need to be overcome to unlock its full potential.

KEYWORDS: mechanical response, proteins, hydration layers, Young modulus, atomic force microscopy, molecular dynamics, antibodies, immunoglobulin

7 he advent of atomic force microscopy $(\mathrm{AFM})^{1}$ stands as a 1 pivotal turning point in nanoscience and nanotechnology. $^{2,3}$ Its ability to operate in almost every environment ${ }^{3,4}$ endows AFM with a great versatility. This ultimately forged its success in many disparate areas, spawning from earthquake dynamics $^{5,6}$ to biological processes. ${ }^{2,7}$ Considering the latter, two major features have driven the interest toward this technique: the first being the ability to image in nearphysiological conditions and the second being the possibility to image cells and biomolecules with a nanometer resolution. $4,8,9$ However, attaining a high resolution on such complex soft systems called for a long and continuous effort to develop a plethora of new imaging modes, ${ }^{2,7}$ each pushing forward a given limitation of the technique. Notwithstanding, and despite its undisputed success, ${ }^{2,4,7,10}$ angstrom/quasi-angstrom reso- lution at the single-protein level has so far remained elusive-a feat long achieved on stiffer substrates. ${ }^{11-14}$

On dense biomolecular assemblies, sub-nanometer resolution can already be readily achieved. ${ }^{8}$ Notwithstanding, here we focus on single-molecule experiments in low-coverage regimes. Despite their inherent difficulties, e.g., higher biomolecular mobility hampering the resolution, they allow us to probe specific biomolecular interactions as well as their

Special Issue: Commemorating NJ Tao

Received: October 26, 2020

Accepted: January 19, 2021

Published: January 27, 2021

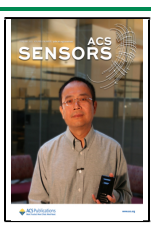


a)

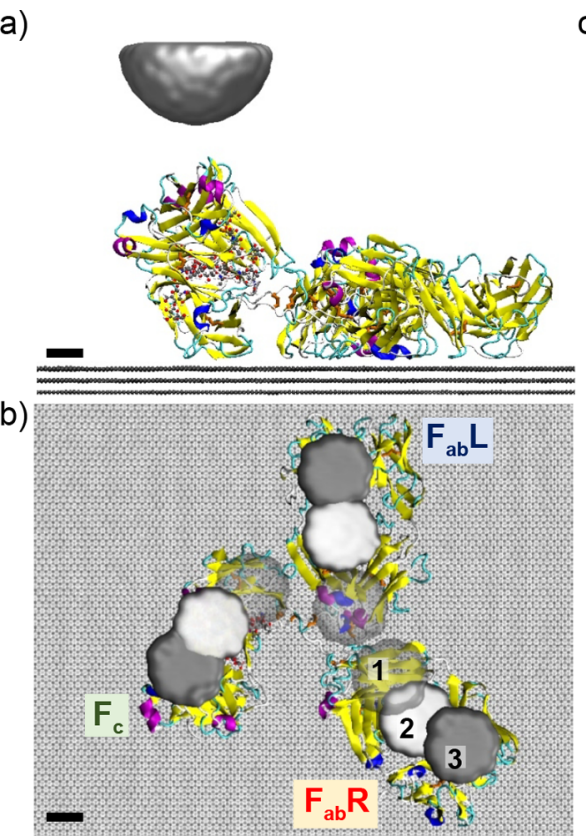

c)

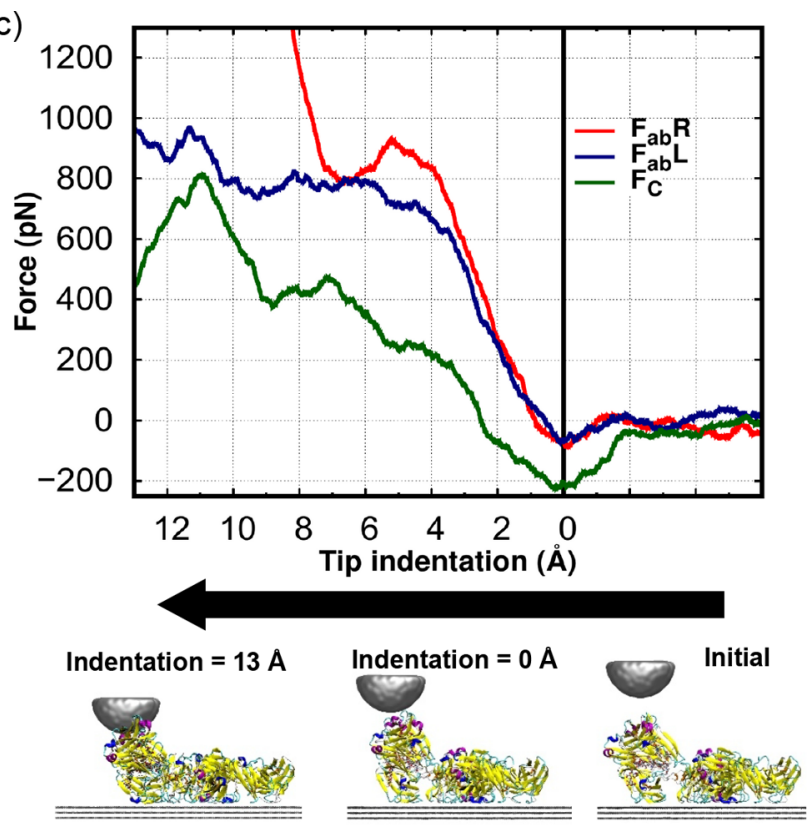

Figure 1. Nanomechanical mapping of an IgG adsorbed on a graphite slab. Side (a) and top views (b) of an IgG adsorbed on a three-layer graphite slab prior to indentation. The notation of the IgG domains and indentation sites is provided in (b). The IgG is represented according to its secondary structure, i.e., $\beta$-sheets (yellow), $\alpha$-helix (purple), $3_{10}$-helix (dark-blue), turns (violet), and random coils (cyan). Graphite is represented with a gray ball-stick model and the diamond tip with a Connolly surface. ${ }^{36}$ (c) Representative force-indentation curves at different sites. Snapshots of different indentation stages are provided in the bottom. Zero indentation is defined upon protein-tip contact and with the onset of a repulsive regime. Scale bar is $1 \mathrm{~nm}$ long.

intrinsic properties, to name a few: single-molecule mechanics, ${ }^{15-17}$ kinetics ${ }^{18,19}$ and specific biomolecular recognition events. $^{20,21}$ Over the past decades single-molecule biophysics emerged as a vibrant research field allowing one to decipher a wide range of complex biological processes. ${ }^{2,4,7,10}$ As a result it follows that a resolution improvement (e.g., through identification of charged, hydrophobic, or mobile regions on a single protein) would most definitely aid in this endeavor, e.g., by improving our understanding of biomolecular processes with an unprecedented level of detail. In this regard, nanomechanical force spectroscopy ${ }^{10,22}$ stands as a promising candidate. Besides topographic information usually gathered during an AFM scan, with this technique we may simultaneously map the forces felt by the tip as we scan. These, being generally more local in nature, could then complement and enhance the spatial resolution of topographic images.

Over 25 years ago, the pioneering experiments of Hansma and co-workers ${ }^{23,24}$ demonstrated AFM's ability to map mechanical properties of soft materials. Although such measurements transcended into a well-accepted characterization tool of the mechanical properties of polymeric surfaces, ${ }^{25}$ at the single-protein level there is still a long road ahead. At this point, an important distinction must be made concerning force spectroscopy methods on single proteins. These can be broadly divided into two classes: one where the molecule is subject to a pulling/extensive force; ${ }^{10,26-32}$ the other where a compression force is applied ${ }^{2,10,22}$ - the latter being the focus of the present work. Among the aforementioned, although not numerous, one may find quite remarkable achievements, including: mapping unfolding events at singleprotein-domain level, ${ }^{33,34}$ and mapping the Young's on a single protein. ${ }^{15,16,35}$ These works showcase how local force mapping provides a much more detailed picture of the protein structure and dynamics, as compared to topography alone.

Although nanomechanical spectroscopy mapping experiments provide valuable information on protein dynamics and mechanical properties, it is far from trivial to relate those with reorganization events occurring inside the protein. For instance, to what extent a macroscopic quantity such as the Young modulus is representative of the deformation processes occurring in a small inhomogeneous object such as a protein? Assuming so, what kind of structural changes regulate this elastic response? Besides the fundamental interest of such questions, they bear an immediate practical application, namely, to gauge the limiting spatial resolution one may attain with such techniques. Still, another relevant question is whether such methods can be used to probe hydration properties of single proteins. This would most certainly provide valuable information, as often biomolecular interactions are triggered either by hydrophobic or electrostatic interactions-both with distinct hydration signatures. All considered, it stands out the importance of complementing experiments with all-atom simulations. This effort would not only provide an atomic understanding of such processes but it would also unveil possible unforeseen phenomena which could result in artifacts in the nanomechanical maps.

Here, we perform all-atom molecular dynamic simulations to simulate the indentation process of a single antibody (the immunoglbulin G) adsorbed on a graphene surface. Our results clearly reveal three different contact regimes, in agreement with previous AFM experiments. ${ }^{22}$ Interestingly, in each regime, the recorded forces probe complementary intrinsic properties of the protein in question. The level of detail of our simulations provides a clear picture of the key atomic reorganization processes occurring in each regime, namely, a detailed understanding on the role of hydrophobic 
a)

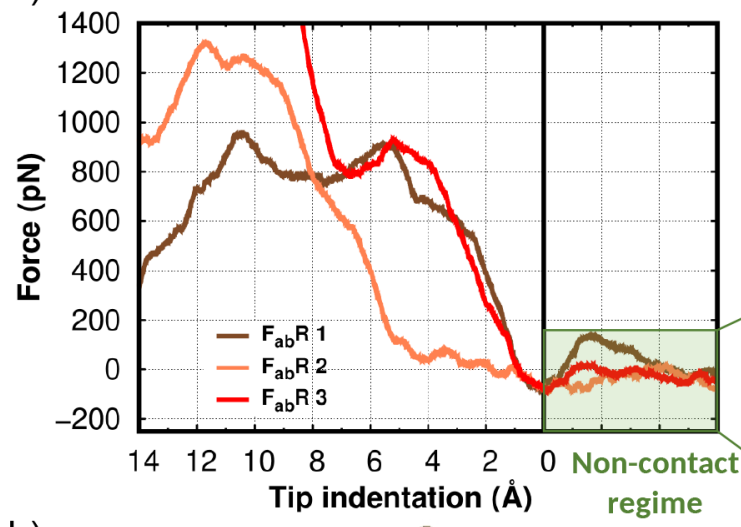

b)

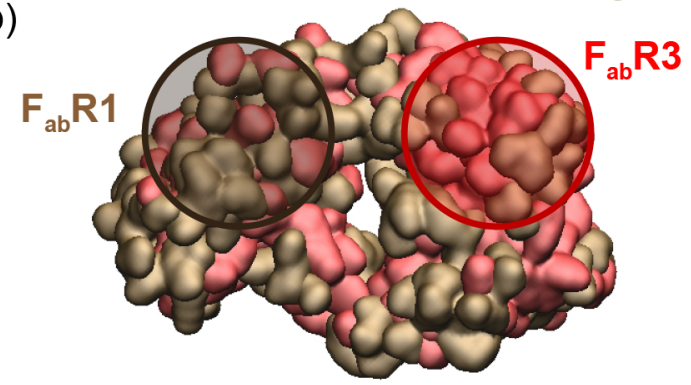

c)

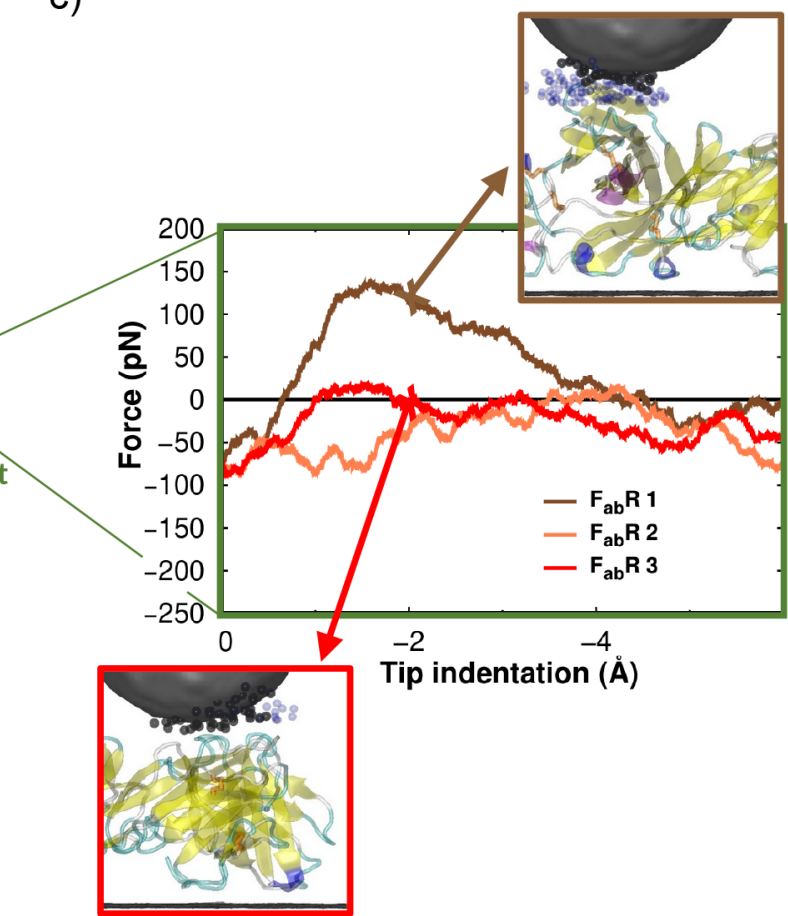

Figure 2. Protein hydration layers detected in the noncontact regime. (a) Force distance curve at different $F_{a b} R$ sites. (b) $F_{a b} R$ represented with a Connolly surface ${ }^{36}$ and colored according to the hydrophobic character, i.e., brown/red for hydrophilic/hydrophobic amino acids, respectively. (b) Zoom in of the force distance curve in the noncontact range. The insets show the presence/lack-of (top/bottom, respectively) a structured water layer over different $\mathrm{F}_{\mathrm{ab}} \mathrm{R}$ sites.

patches on hydration layers and related hydration forces; the global character of elastic mechanical deformation limiting the spatial resolution to mechanically decoupled domains; and the importance of spurious protein mobility events in the interpretation of flexibility and critical unfolding force of protein domains. In this way, besides complementing previous experiments with an atomic detailed picture of such processes, we also provide novel insights on how different force regimes can enhance spatial resolution with the hope to stimulate further experiments.

\section{RESULTS AND DISCUSSION}

For the substrate, we consider an IgG antibody adsorbed flaton over a three-layer graphene surface (see Figure 1 and Methods). Previous works ${ }^{37,38}$ have shown good agreement between adsorption configurations imaged with amplitude modulation (AM) AFM and all-atom molecular dynamics (MD) simulations. This was corroborated in various ways, to name a few: similar interdomain distances (i.e., $\mathrm{F}_{\mathrm{ab}}-\mathrm{F}_{\mathrm{c}}, \mathrm{F}_{\mathrm{ab}}-$ $\left.\mathrm{F}_{\mathrm{ab}}\right)$; preservation of secondary structure and bioactivity of the $\mathrm{F}_{\mathrm{ab}}$ sites; and that the $\mathrm{F}_{\mathrm{c}}$ domain is systematically higher than the smaller $\mathrm{F}_{\mathrm{ab}}$ sites. Concerning the former, it may be best understood in light of Figure 1a. There, it is also apparent that glycan heteropolymers in the center of the $\mathrm{F}_{\mathrm{c}}$ domain create a hollow region inside its structure. One may thus expect that by being less compact, $F_{c}$ domains could be readily identified in an AFM nanomechanical map ${ }^{22}$ as the most soft domain. To test this hypothesis, we indented on three different sites in each IgG domain (see Figure $1 b$ ). Representative force-indentation curves are shown in Figure 1c. As anticipated from the protein structure, we observe that $F_{c}$ has a softer mechanical response than $\mathrm{F}_{\mathrm{ab}}$ domains. Comparing our results with the pioneering experiments of Martinez-Martin et al. ${ }^{15}$ reveals a qualitative agreement between them, namely, that $F_{c}$ is softer than $F_{a b}$ domains. Note, however, that these experiments ${ }^{15}$ where performed for a different protein (IgM, which is a pentameric structure composed by five IgG monomers) deposited on a different substrate (mica). In this context emerges the field of nanomechanical force spectroscopy, ${ }^{15,22,29,35,39}$ where AFM experiments are used to map the elasticity of such complex systems at a local level. Yet, it remains unclear the origin of such elastic behavior and to what extent one may extrapolate a macroscopic quantity, such as the Young modulus, to inhomogeneous objects such as single proteins. To address this question, while unveiling the limits of the lateral resolution of such techniques as well as other phenomena (e.g., water structuring near the proteins), we now explore the atomic detail of our MD simulations in the three contact regimes: noncontact regime, elastic regime, and high-strain regime.

Noncontact Regime. AFM has been successfully employed to image hydration layers formed over a wealth of different substrates including the following: inorganic materials, ${ }^{40,41}$ molecules, ${ }^{42-44}$ protein assemblies, ${ }^{45,46}$ and also over double-stranded (ds) DNA molecules. ${ }^{47}$ Surprisingly, this feat has remained elusive for single proteins. The importance can hardly be overstated as often biomolecular recognition/activity relies on hydrophilic/phobic interactions. ${ }^{48,49}$ With the aim of identifying hydrophobic/hydrophilic patches of the protein, we now focus on the initial indentation stage (i.e., prior to contact). For that purpose, we show in Figure 2a two forceindentation curves obtained in the same domain, i.e., $F_{a b} R$. It is apparent that before contact (event marked as zero indentation), $\mathrm{F}_{\mathrm{ab}} \mathrm{R} 1$ and $\mathrm{F}_{\mathrm{ab}} \mathrm{R} 3$ behave very differently, with one showing a repulsive force peak and the other lacking such feature. This difference can be readily understood in light of 
a)

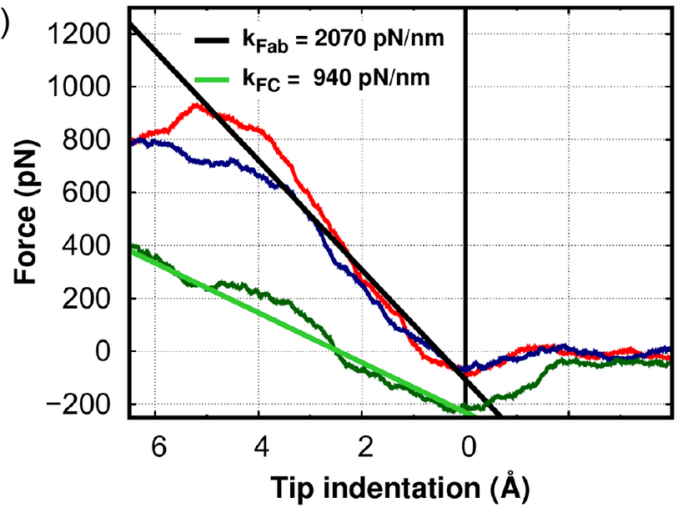

c)

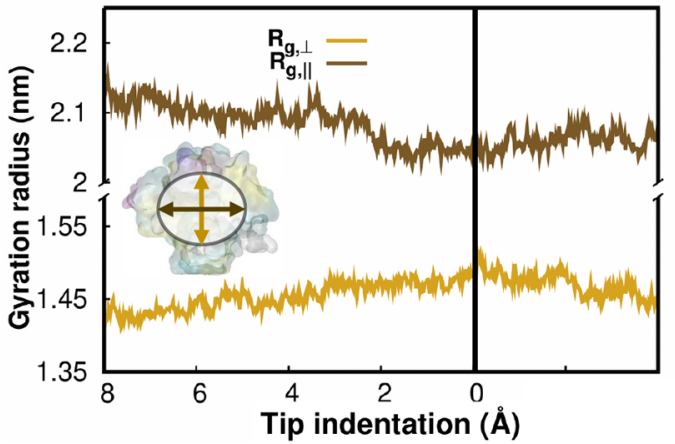

b)

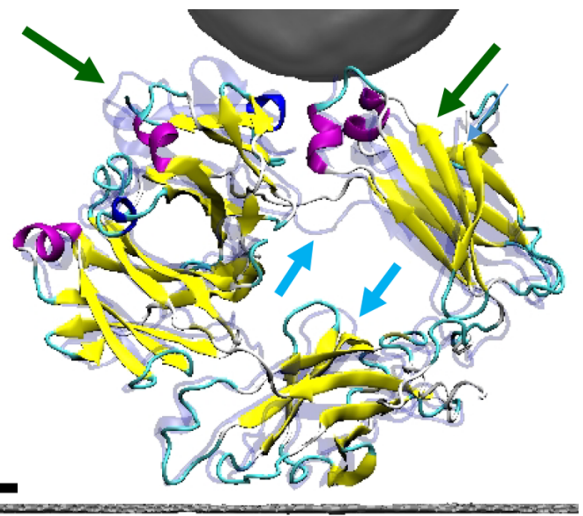

d)

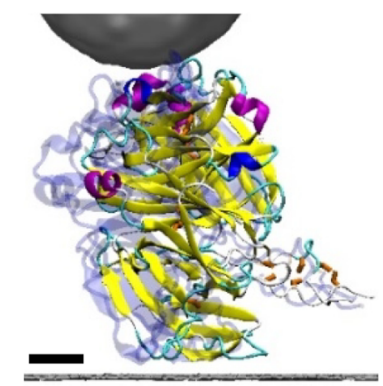

Figure 3. Global deformation of the $\mathrm{F}_{\mathrm{c}}$ domain in the elastic regime. (a) Representative force distance curves of the three domains. The associated stiffness is obtained from the linear fits (shown in black and light green). (b) Side view before/during elastic deformation represented as transparent/opaque. Green arrows highlight rigid displacement of well-defined secondary structures. Cyan arrows highlight flexible unstructured domains deformed upon indentation. (c) Parallel/perpendicular gyration radius represented in brown/light-orange. The inset provides a visual interpretation of the data, e.g., the parallel component being a expansion/contraction of the protein in the plane parallel to the surface. (d) Side view of images in panel $\mathrm{b}$ highlighting a deformation penetration depth $>4 \mathrm{~nm}$. Scale bar is $1 \mathrm{~nm}$ long.

the hydrophilic character of each site; see Figure $2 b$. In particular, we observe that the first site is predominantly composed by hydrophilic amino acids while the other by hydrophobic ones.

Focusing on $\mathrm{F}_{\mathrm{ab}} \mathrm{R} 1$, we realize that the forces required to break the hydration layer (shown in Figure $2 c$ ) are on the same range as those experimentally measured on dsDNA, ${ }^{47}$ i.e., 100 $\mathrm{pN}$. Here, the hydration layer owes its presence to the large amount of hydrophilic amino acids as shown in Figure $2 \mathrm{~b}$. They have an important biological role on the IgG activity. This is apparent when noting that $\mathrm{F}_{\mathrm{ab}} \mathrm{R} 1$ is positioned near the hinge region, which is responsible for regulating the large oscillations of the $\mathrm{F}_{\mathrm{ab}}$ in solution ${ }^{50}$ as well as on-surface motion. Considering the former, it has been shown that such mobility is key in assisting the IgG to walk over antigenic surfaces. ${ }^{19}$ The hydrophilic character of this site favors solvent exposition with respect to nonspecific interaction with neighboring domains (e.g., $F_{c}$ or the other $F_{a b}$ ), which ultimately regulates $\mathrm{IgG}$ bioactivity. ${ }^{19,50}$

The $F_{a b} R 3$ is located at the antigen binding site where most amino acids display a hydrophobic character; see Figure $2 \mathrm{~b}$. This is a key property for docking, activation, and signaling of antigen-binding fragments at large, ${ }^{48,49,51}$ present both in immunoglobulins as well as in $\mathrm{T} / \mathrm{B}$ cell receptors. Consequently, at this site, nonspecific protein-tip interactions are favored over hydration and thus no force is required to displace water molecules surrounding these amino acids; see Figure $2 \mathrm{c}$. In fact, we observe that once water is depleted, an attractive force is felt by the tip-signaled as negative force values. Interestingly, this attractive force depends not only on the amount of hydrophobic amino acids but also on the relative mobility of those. In Supporting Information Figure $S 1$, we provide an example where, upon indentation, a local spontaneous reorganization at the $\mathrm{F}_{\mathrm{c}} 2$ site occurs resulting in a jump-to-contact of the protein toward the tip. This process is signaled via an abnormally pronounced attractive regime as shown in Figure S1. The slope of the force curve in the attractive regime relates to the stiffness of this local reorganization process. Although common cantilevers are too soft to detect such deformation-as they would jump to contact-recently developed tuning fork sensors $^{52}$ are in principle capable of probing such events.

In the noncontact regime our simulations show that one could in principle distinguish hydrophilic from hydrophobic regions within a protein domain (on the sub-5 nm scale) at the single-molecule level. The forces required to break hydration layers, although small, are within the experimental resolution. ${ }^{47}$ Additionally, our simulations unveil how the soft character of the protein can allow for spontaneous local reorganization events, signaled as a pronounced adhesion force, which could relate to dissipation processes as discussed elsewhere. ${ }^{22}$

Elastic Regime. In the low-strain regime (i.e., <10\%), the force increases linearly with indentation as in an elastic deformation process (see Figure 3a). A linear fit to the force curve allows one to compute the Young modulus or, equivalently, the protein elasticity at different indentation sites. As show in Figure S2, the Young modulus is almost constant in each domain. This suggests that the elastic response is a global property of these domains and is not controlled by the local area around the indentation site. This is 

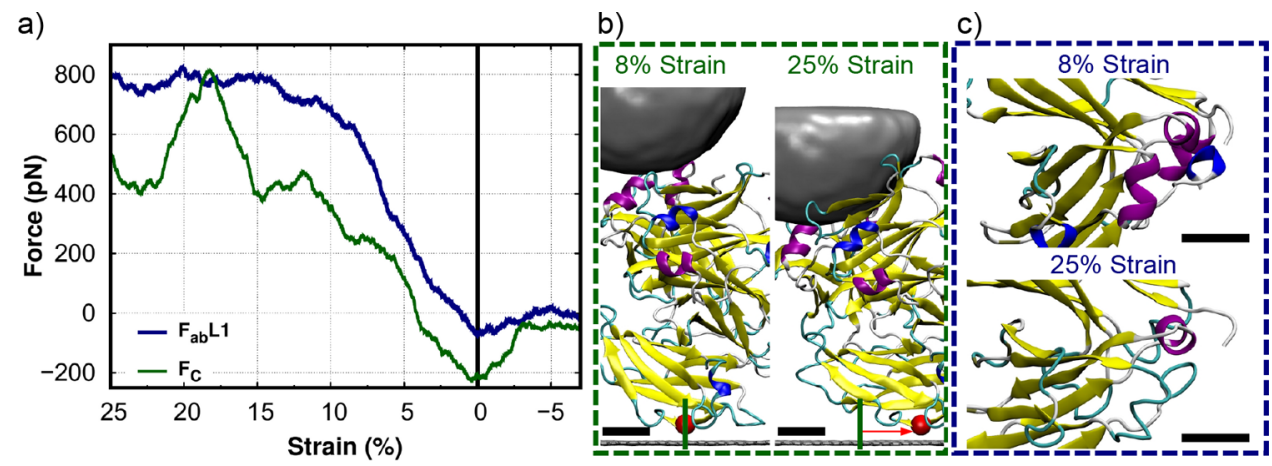

Figure 4. High-strain deformation regime: sliding and unfolding. (a) Force distance curve at $\mathrm{F}_{\mathrm{ab}} \mathrm{L} 1$ (in blue) and $\mathrm{F}_{\mathrm{c}} 2$ sites. (b) Sliding event occurring during indentation on the $\mathrm{F}_{\mathrm{c}} 2$ site. An $\alpha$ carbon of the $\operatorname{IgG}$ is represented in red, so we can better appreciate the displacement event with respect to fixed reference highlighted as a green line. (c) Area under the tip while indenting on the $\mathrm{F}_{\mathrm{ab}} \mathrm{L} 1$ site. Protein representation is identical to Figure 1. Scale bar is $1 \mathrm{~nm}$ long.

in line with a sensible argument that mechanical elasticity can only change from one region to another (as in $\mathrm{F}_{\mathrm{c}} / \mathrm{F}_{\mathrm{ab}}$ ) provided that they are mechanically decoupled, e.g., linked by an unstructured chain of amino acids (random coil/turn). Additionally, the consistency of the stiffness values obtained for the two different $\mathrm{F}_{\mathrm{ab}}$ domains (see Figure 3a and Figure S2a) not only corroborates this argument-as they are structurally identical-but also unravels that elasticity is insensitive to the adsorption configuration up to certain degree. This follows from the fact that $\mathrm{F}_{\mathrm{ab}} \mathrm{L}$ and $\mathrm{F}_{\mathrm{ab}} \mathrm{R}$ have similar stiffness values although adsorbed in a flipped conformation with respect to the adsorption/surface plane. ${ }^{37}$ At last, and perhaps the most promising result, we observe that different domains, i.e., $\mathrm{F}_{\mathrm{ab}}$ and $\mathrm{F}_{\mathcal{O}}$ have different stiffness values, i.e., 2.1 and $0.9 \mathrm{~N} / \mathrm{m}$, respectively. Not only is this result is in line with the fact that $F_{c}$ is a hollower structure but it suggests that one could in principle use the flexibility map to enhance AFM topographic resolution. In fact, such differential flexibility was previously reported on an alike protein using biomodal AFM experiments. ${ }^{15}$ More recently, this submolecular resolution of nanomechanical mapping was pushed even further, now allowing one to resolve major/minor grooves of a single DNA molecule. ${ }^{47}$ However, and despite the authors' insight, ${ }^{15}$ the results were based on two nontrivial assumptions that lacked validation. The first being the possibility to define a macroscopic quantity as the Young modulus in a small and inhomogeneous object such as a protein. The second was the assumption that the Young modulus is well-defined even at a local level in the protein, e.g., in its domains.

In order to cast light on how strain is accommodated inside the protein, we now inspect the $\mathrm{F}_{\mathrm{c}} 2$ indentation results-note that similar findings are obtained on other domains. In Figure $3 b$, we observe that the most affected region concerns the amino acids in direct contact with the tip. However, the indentation induced deformation propagates throughout the protein penetrating as deep as $4 \mathrm{~nm}$ from the indentation site; see Figure $3 \mathrm{~d}$. This global concerted motion of the protein is, to a large extent, mediated by the most flexible regions inside each domain. These are the regions without a well-defined secondary structure such as turns and random coils. For example, in Figure $3 \mathrm{~b}$ we observe that the structure of antiparallel $\beta$-sheets is preserved (highlighted with green arrow in Figure $3 b$ ) differing only from the un-indented protein by a rigid downward shift. This motion and the structure preservation is regulated by the most flexible regions (highlighted with cyan arrows in Figure 3b), which allows redistribution of the stress throughout the protein, ultimately resulting in a global deformation. This rationalizes why similar stiffness values are obtained at different sites within the same domain, as shown in Figure S2. This global deformation is particularly visible in the gyration radius shown in Figure $3 \mathrm{c}$. There we observe that upon indentation, the protein compression is accompanied by an expansion in the plane perpendicular to indentation, i.e., the plane defined by the surface.

One may thus conclude that the Young modulus is a welldefined property within each domain as it reflects a global deformation mediated by the most flexible regions (i.e., random coils and turns). These regions can accommodate strain without disrupting the hydrogen-bond network, thus allowing swift recovery of the initial configuration once strain is released. Consequently, we did not observe changes on the secondary structure at these strains. Moreover, it is interesting to note that the elasticity measured in this or in alike experiments ${ }^{15,22,35}$ is rather small as compared to the hydrogen bonds responsible for the structural stability of the protein. A conservative estimate for such stiffness is of $3 \mathrm{~N} / \mathrm{m}$ per $\mathrm{H}$ bond. $^{53-55}$ Drawing from a mechanical analogue based on springs, either in series or parallel, we see that although the stiffness sum is parallel in a single strand (with more than five hydrogen bonds per strand), these are in turn to be summed with the adjacent strands as springs in series; see Figure S3. Thus, the higher number of turns/ $\beta$-strands being deformed by the tip, the higher is the compliance (i.e., lower stiffness) of the protein. As a result, it follows a positive correlation between topography and flexibility. This is seen in our simulations when comparing $\mathrm{F}_{\mathrm{c}}$ and $\mathrm{F}_{\mathrm{ab}}$ domains; i.e., the higher is the domain (or, equivalently, the larger the number of turns), the lower is the elasticity. Although being rather simplistic to account for the full complexity of the problem at hand (neglecting the relative orientation of the strands and the importance of mixed secondary structures, among others), this analysis puts in evidence that, in this kind of measurements, little information may be drawn concerning hydrogen-bond dynamics (contrary to other force-spectroscopy techniques ${ }^{31,32,56-59}$ ). Moreover, it provides a simple interpretation on the correlation between topography and flexibility in very specific cases. ${ }^{15}$

High-Strains Regime: Unfolding and Plastic Deformation. At high deformations (strains of $15 \%$ or higher), one of two deformation mechanisms are observed: either major off- 
tip sliding, or unfolding events. Concerning the first, such events may be readily detected as a non-monotonic behavior of the force; see Figure 4a. Although protein mobility seems eminent almost throughout the whole repulsive regime, it becomes prevalent when protein deformation surpasses $12 \%$ (note that $F_{a b}$ and $F_{c}$ average heights are 4.3 and $6.3 \mathrm{~nm}$, respectively). At such high deformations, the protein releases strain by sliding as much as $1.5 \mathrm{~nm}$; see Figure $4 \mathrm{~b}$. This results in a force decrease, as shown in Figure $4 a$, followed by its increase-in some sort of complex stick-slip motion. Concerning unfolding events, they are characterized by a plateau in the force curve as indentation proceeds; see Figure 4 a. This could be perceived as a plastic deformation regime as reported in previous experiments. ${ }^{22,35}$ As shown in Figure 4c, during this process, the protein undergoes an unfolding event where the loss in the secondary structure follows from the breaking of weak hydrogen bonds-a process which can be viewed as irreversible (plastic) at the time scales of the indentation process.

Although, in general, all-atom MD simulations are unable to reach the slow indentation speeds used in AFM experiments (further discussion is provided in the following section), one may argue that they can serve as an upper limit guide in such a high-strain regime. On the one hand, sampling less the slow degrees of freedom, we are overestimating the force required to deform the protein. ${ }^{54,60}$ On the other hand, when major reorganization events such as sliding or unfolding occur in the simulations at a given strain, then it is sensible to assume that, in experiments, they will occur at lower or equal strains-given the larger exposition time of the protein to the force exerted by the tip. Concerning protein unfolding, it is well-known that the higher is the speed, the higher is the yield strain of the protein. ${ }^{54}$ Then, it follows that the simulated unfolding strain provides an upper limit beyond which protein unfolding is very likely to occur at the slow indentation speeds used in experiments. All together our simulations allow us to propose the following rules of thumb for indentation at high strains: (a) For globular proteins of similar size as the IgG, beyond $15 \%$ strain we are no longer in an elastic deformation regime; (b) unfolding is characterized by two factors, one being an alteration of the topographic profile and the second being a stabilization of the force during indentation; (c) if major sliding events are to occur (often in regions where the ratio between contact area and the protein volume is small such as in the $\mathrm{F}_{\mathrm{c}}$ domain), such events may be identified as a nonmonotonic behavior of the force curve, at variance with the unfolding case.

Comparing the aforementioned predictions with previous experiments, ${ }^{35}$ we find a discrepancy for the onset of nonelastic protein deformation. Those authors suggest that elastic deformation could endure strains of up to $40 \%$, which would correspond to an indentation of $2.4 \mathrm{~nm}$ on the $\mathrm{F}_{\mathrm{c}}$ domain. According to our simulations, the IgG could hardly accommodate such levels of strain without slipping away or undergoing a major unfolding event. This is in line with other studies $^{54}$ showing that, beyond a strain of $15 \%$, proteins with varied secondary structures undergo unfolding events. It is possible that the difference between our results and previous experiments ${ }^{35}$ follow from different indentation speeds, protein sizes, or supporting substrate. Thus, further works are needed to clarify this issue. However, a plausible reconciliation might be achieved if we consider that protein mobility events can be misinterpreted as a soft elastic deformation. In such on-surface mobility process, the protein retains its equilibrium height in a subsequent topography imaging (typically a fingerprint for the onset of plastic deformation). However, this is one of many possible scenarios that could account for the observed difference. To address this issue, we propose further experiments to measure the strain threshold in the low surface coverage regime simulated here and in a high-coverage regime, which would mitigate the role of the mobility.

Simulation Challenges: Bridging Experiments/Simulations Time Scales. From a simulation standpoint, in order to mimic the indentation process as in AFM experiments, it stands clear the importance of incorporating both the surface supporting the protein as well as an explicit description of the water molecules. This follows from two major reasons: the first being the importance of protein-surface adhesion in regulating sliding mechanisms as well as mechanical deformation; ${ }^{22}$ and the second concerns the importance of hydrophobic/hydrophilic interactions as well as the role of hydration layers in the noncontact regime. With regard to the accuracy of different solvation methods, we have shown ${ }^{38,61}$ that implicit methods-commonly used in protein adsorption processes-result in a systematic spurious protein unfolding during adsorption. This follows from a misrepresentation of entropic effects of the solvent and an unbalanced electrostatic force when protein-surface start to contact. Concerning protein adsorption simulations, we have shown how MD simulations allowed us to obtain a good agreement with AFM experiments in varied conditions (different proteins, ${ }^{37,61}$ on hydrophilic/phobic surfaces, ${ }^{62,63}$ with and without step edges ${ }^{64}$ and in free/forced adsorption regimes ${ }^{61}$ ), while providing an atomic detail of this process.

Molecular dynamics simulations are a powerful technique to simulate force spectroscopy experiments, ${ }^{12-14}$ e.g., allowing one to unravel the atomic mechanisms for chemical identification in liquids. ${ }^{12}$ However, the duration of such simulations is often limited to time scales that are much shorter than in experiments. One major limitation of the simulations here reported is the indentation speed $(0.1 \mathrm{~m} / \mathrm{s})$ as they largely surpass the ones used in experiments, i.e., $10^{-4} \mathrm{~m} /$ s. Preliminary experimental results for $\operatorname{IgG}^{65}$ indentation on mica estimate a stiffness of $0.25 \mathrm{~N} / \mathrm{m}$. When comparing to our simulations, we realize that we are overestimating the mechanical elasticity by a factor of 6 . This overestimation at higher loading rates is in agreement with Bell-Evans models. ${ }^{66,67}$ Moreover, this is also in line with other forcespectroscopy measurements/simulations ${ }^{68}$ as well as protein unfolding events. ${ }^{54}$ This overestimation follows from a poor representation of stochastic fluctuations of the protein during the indentation process. ${ }^{60,68}$ However, a brute force approach to overcome this limitation, i.e., matching experiments and simulation times, would render such simulations unfeasible as it would require millisecond long simulations. Although one may correct the force values using different models, ${ }^{60,68}$ these are often reserved for bond breaking processes, thus significantly different from global protein reorganization events. Despite the overestimation of the force, the trajectories thus obtained can provide a realistic representation of the deformation processes taking place during the indentation. This follows from the fact that the major structural differences observed at different deformation speeds happen at high strains, ${ }^{54}$ where protein unfolding starts to occur. Therefore, as long as the secondary structure is preserved, the structural 
a)

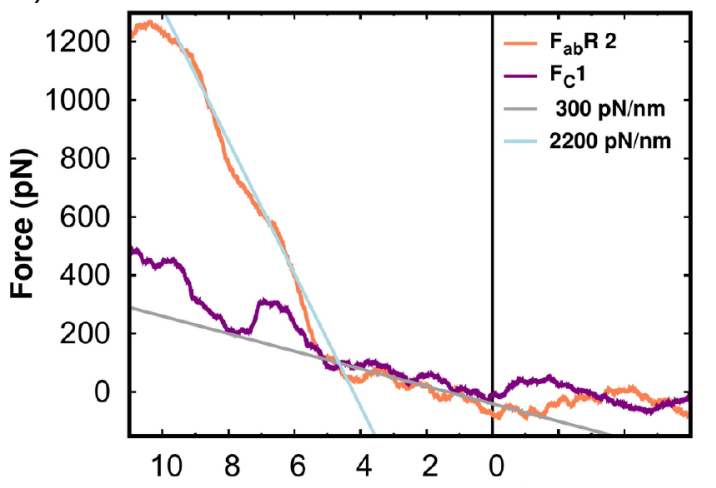

c)

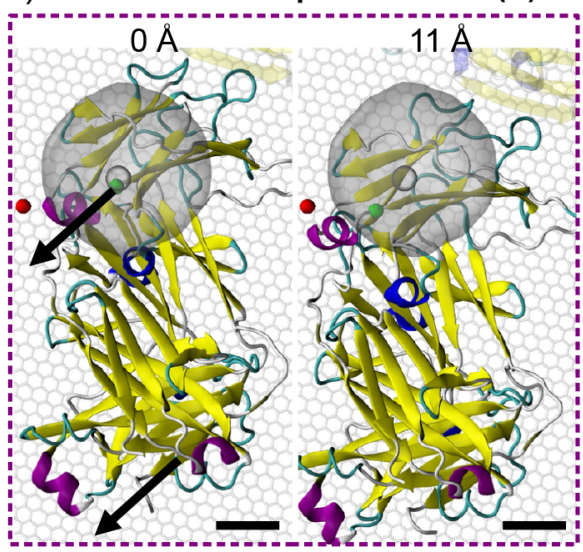

b)

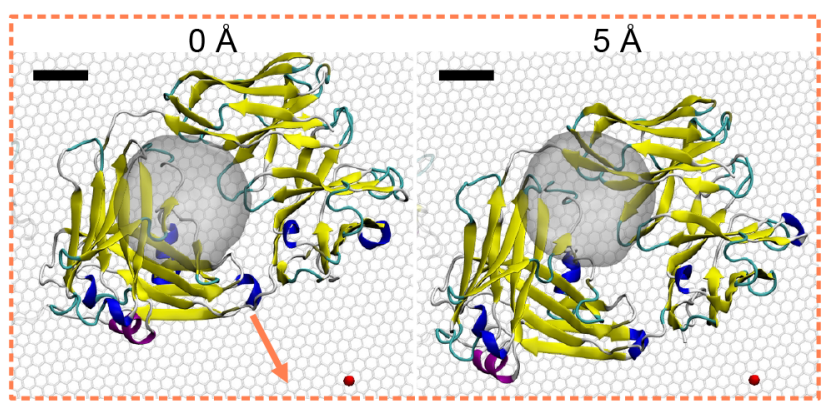

e)

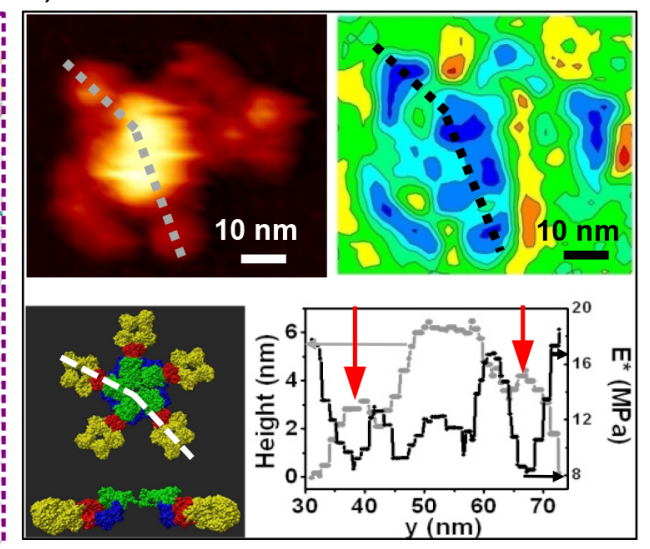

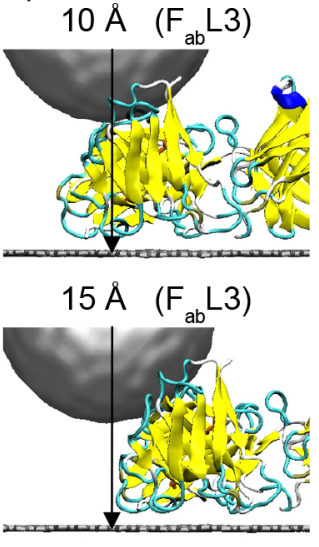

Figure 5. Spurious elastic softening resulting from protein mobility. (a) Force curves at $\mathrm{F}_{\mathrm{ab}} \mathrm{R} 2$ and $\mathrm{F}_{\mathrm{c}} 1$ indentation sites. (b, c) Snapshots at different indentation depths showing on-surface protein displacement at the $\mathrm{F}_{\mathrm{ab}} \mathrm{R} 2(\mathrm{~b})$ and $\mathrm{F}_{\mathrm{c}} 1$ (c) sites. Arrows depict the direction of motion, and red/green spheres provide reference points at the surface/protein. (d) From left to right, top to bottom: topography, elastic map, protein structure, and topography/flexibility profiles of an IgM on mica obtained using bimodal AFM imaging. ${ }^{15}$ In the profile image, topography/flexibility are represented in gray/black. The red arrows highlight topography/flexibility maxima/minima at $\mathrm{F}_{\mathrm{ab}}$ sites of the IgM. (e) Tip induced sliding at the edge of a $\mathrm{F}_{\mathrm{ab}}$ obtained in our simulations at the $\mathrm{F}_{\mathrm{ab}} \mathrm{L}$ 3. Scale bar is $1 \mathrm{~nm}$ long. Reproduced from ref 15 with permission of The American Physical Society. Copyright 2011.

analysis derived is expected to resemble the one obtained at lower indentation speeds.

An alternative approach to a brute-force speed matching approach can be provided by constant force molecular simulations ${ }^{69,70}$ (CFMD). Recent works have shown this protocol is able to compute the elastic parameters of nucleic acids in quantitative agreement with experimental results. ${ }^{69,70}$ The protocol is compatible with nanomechanical force spectroscopy mapping and can be summarized as follows. Starting from a tip-protein contact conformation, we start multiple $\mathrm{MD}$ runs in parallel. In each run the force applied to the tip is kept constant at different selected values. Once the system is finally in equilibrium, we then have different pairs of force-distance values. In this way we are able to equilibrate the trajectories for each constant force value for periods as long as a microsecond. As a result each pair of force/indentation can be viewed as a static picture of the indentation process, which would be more representative of the slow indentation speeds used in the experiments. Note, however, that this simulation protocol relies on the simple assumption that we know a priori the shape of the force curve. Then, instead of sampling all indentation depths we focus on a few selected points of force versus indentation which are well-converged. This fact highlights the importance of the results shown in the present work, which provide a lively picture of the whole indentation process-a feature missing in the aforementioned protocol.
Thus, our results constitute a first step toward an atomic detailed understanding of the complex phenomena occurring during the indentation. Future endeavors relying on the aforementioned CFMD protocol could provide a better quantitative agreement with available experiments at similar computational cost.

At last, another aspect which may have an impact on the measured forces is the tip size, shape, and composition. In this work we considered a tip with properties that are consistent with commercially available ultrasharp (radius of $2 \mathrm{~nm}$ ) diamond tips. However, it would be interesting to test the influence of other commonly used tips. Although we do not expect that such variations will change our general conclusions, the nominal forces obtained may vary as a result.

Experimental Challenges: Protein Mobility and Spatial Resolution. In what follows, we discuss the two important aspects revealed by our simulations that could aid in the interpretation of flexibility maps as measured by AFM, namely, protein mobility and the spatial resolution of such measurements.

Protein Mobility. We often observe that, even at early indentation stages, protein sliding can result in forcedisplacement curves that carry little information about its mechanical/structural properties. In Figure 5, we provide two examples obtained at small strains $(<10 \%)$. The first, and the easiest to identify, is the one obtained while indenting in the 
middle of the $\mathrm{F}_{\mathrm{ab}}$ domain, namely, in the $\mathrm{F}_{\mathrm{ab}} \mathrm{R} 2$ site. During the first $0.6 \mathrm{~nm}$ of indentation, the force increases at a much lower rate than the other sites; i.e., it seems to be much softer than the other sites (see Figure 5a). A close inspection of the trajectory shows that during this period we are not indenting the protein, as, instead, the $F_{a b} R$ is sliding on the surface (see Figure 5b). Only after a stable tip-protein contact is formed, one starts to mechanically deform the domain and we recover similar stiffness values as in other sites of the domain (see Figure 5a). The second example is far less trivial to identify from the force curve alone and, thus, could pass unnoticed in experiments. As we indent the $\mathrm{F}_{\mathrm{c}} 1$, the force increases steadily, although at a rate much smaller than that of any other site (see Figure 5a). Once more, this one could mislead us to think that the $F_{c} 1$ site is the softest site in the $F_{c}$ domain. However, from the trajectory, we realize that this indentation instead of deforming the protein is pushing it away from the indentation site in a major displacement event (see Figure 5c). The aforementioned examples show how mobility events can dramatically affect our elasticity measurements. Moreover, the on-surface motion, although being sufficiently large (0.1$0.5 \mathrm{~nm}$ ) to affect such measurements, they are small enough to be interpreted as a product of thermal drift.

In Figure 5d, we show an elasticity map of a similar protein (IgM on mica) obtained using bimodal AFM. ${ }^{15}$ From the topography, it is apparent the large mobility of the $\mathrm{F}_{\mathrm{ab}}$ domains sitting at the edge of the protein. Moreover, the topography (shown in gray) and the flexibility profiles (shown in black) reveal a mismatch. This difference is particularly evident at $y=$ $37 \mathrm{~nm}$, which corresponds to a $\mathrm{F}_{\mathrm{ab}}$ domain (highlighted with a red arrow in Figure 5d). At this site, the topography profile shows a local height maximum, whereas the flexibility profile shows a sharp drop of the Young modulus. In light of the present simulations, a plausible interpretation is that, at $\mathrm{F}_{\mathrm{ab}}$ edges, indentation forces the fragment to slide, as we have observed in the $\mathrm{F}_{\mathrm{ab}} \mathrm{L} 3$ site- - see Figure 5e. As a result of such sliding, we obtain an apparent, abrupt softening of the last half of the $\mathrm{F}_{\mathrm{ab}}$ domain. A similar observation can be made for the $\mathrm{F}_{\mathrm{ab}}$ sitting at the opposite edge $(y=65 \mathrm{~nm})$. This mobility is also apparent in the corresponding flexibility map. In particular, this map does not have the same symmetry as the molecular structure (i.e., a 5-fold symmetry).

Spatial Resolution of Flexibility Maps. In the elastic regime, our $\mathrm{MD}$ simulations showed similar elasticity for different sites in the same domain; see Figure S2. Additionally, the atomic trajectories reveal that mechanical elasticity is related to a global domain deformation, i.e., not a local one. Therefore, the spatial resolution of flexibility maps is limited to the number of mechanically decoupled domains. Although it is not trivial to infer a priori how many of such domains there are on a given protein, the topographic images and a detailed understanding of the protein structure can provide clues for such assignment. Concerning the first, mechanically decoupled domains give rise to different adsorption geometries. Taking the IgG as an example, ${ }^{37}$ some topographic images show $\mathrm{F}_{\mathrm{ab}}$ closer and some others with $\mathrm{F}_{\mathrm{ab}}$ far apart. Thus, it follows that their motion is decoupled and they may move freely with respect to each other. Concerning the second, generally speaking, regions of the protein with undefined secondary structure (e.g., random coils or turns) constitute elements of enhanced flexibility. These elements provide a means to decouple the motion of different subdomains without breaking hydrogen bonds. This is seen in the IgG at the hinge regions but also on other globular proteins such as the albumin. ${ }^{61}$ At last, it should be noted that, at the edges of small domains such as the $F_{a b}$, it is more likely that the tip indentation will induce motion thus introducing artifacts.

Simultaneous measurement of flexibility and topography in AFM experiments provides a valuable tool to better resolve these complex structures on a surface. The importance can be better realized when tracing a parallel with AFM imaging of nucleic acids which has largely expanded our understanding of a wealth of biological processes ${ }^{71}$ ranging from DNA compaction $^{72}$ to specific protein interactions. ${ }^{20,21}$ Yet, such success builds upon a long and continuous effort in the development of a wide range of DNA immobilization techniques, some focusing on DNA-protein interactions ${ }^{21}$ and others in DNA structural or dynamic properties. ${ }^{73-75}$ Nowadays, resolution in these systems is such that one may routinely resolve minor/major grooves of nucleic acids on both the topography ${ }^{76-78}$ and the stiffness maps. ${ }^{39}$ Perhaps more astonishingly, one may also resolve hydration layers formed over DNA molecules. ${ }^{47}$ We expect that a similar endeavor in the development of specific immobilization protocols on single proteins would help to mitigate protein mobility artifacts.

\section{CONCLUSIONS}

In this work, we use all-atom MD simulations to perform nanomechanical force spectroscopy mapping on a single immunoglobulin $\mathrm{G}$ protein adsorbed on a three-layer graphene surface. Our simulations show three distinct contact regimes: noncontact, elastic, and a high-strain regime.

In the high-strain regime $(>15 \%)$, the protein either starts to unfold or simply slides away from under the tip. The $>15 \%$ strain threshold, although consistent with typical unfolding strains, differs from previous AFM estimates ${ }^{35}$ which revealed a much higher unfolding resilience (up to strains of $>40 \%$ ). Here, we reconciled these two results with the hypothesis that, at such high strains, mobility events, which are hard to discern from the elastic regime, dominate the mechanical response. This interpretation is based on two facts: sliding events of the protein do not deform it permanently, and they display as part of an apparently elastic regime in the force curve. In order to corroborate this hypothesis, further work is needed. Of particular relevance, and readily feasible, would be contrasting AFM experiments on single molecules with AFM measurements on compact protein assemblies ${ }^{28,29}$ where mobility is hampered.

The elastic regime can be identified in the force curve as the deformation comprised between the onset of the repulsive regime and a $10 \%$ strain. In the pioneering work of MartinezMartin et al., ${ }^{15}$ they were able to measure a Young modulus of different immunoglobulin $M$ sites using bimodal AFM. Yet, owing to the lack of atomic detail of such experiments, it was unclear whether this Young modulus related to an elastic deformation, and, perhaps most importantly, what the limiting spatial resolution one may attain in such flexibility maps was. Our MD simulations show that, for small strains, the protein deforms elastically, and thus, the Young modulus is welldefined despite the inhomogeneous character and small size of a protein. This deformation occurs without loss of the secondary structure and is regulated by flexible regions present in each domain (typically unstructured random coils/turns). Moreover, we show that deformation can penetrate as deep as $4 \mathrm{~nm}$ into the protein structure and it involves a global collective motion of all atoms within each domain. Thus, it 
follows that the limiting spatial resolution is given by the number of mechanically decoupled domains. In this sense, our results put on firm ground the conclusions derived from Martinez-Martin et al. ${ }^{15}$ and demonstrate how force spectroscopy is a versatile tool to map mechanically decoupled domains within a protein. It is noteworthy to remark that global elastic deformation is obtained regardless of the fact that we used a very sharp tip $(R=2 \mathrm{~nm})$ - thus suggesting a minor role of the tip radius.

Concerning the noncontact regime, we are able to identify hydrophilic/hydrophobic patches over a same domain. Therefore, this is the regime where the force is most sensitive to the local environment and could best complement topography in order to enhance the spatial resolution. Moreover, besides being non-invasive, it was the least affected by thermal fluctuations and protein mobility. The force range at which the hydration layer is broken is comparable with the hydration forces measured on a single DNA molecule, ${ }^{47}$ showing that such hydration forces are within the current experimental accuracy. Additionally, at certain sites of each domain, we found exceptionally flexible regions that spontaneously jumped to contact the tip-a process accompanied by an abnormally large attractive regime. While such regimes may be hardly explored with soft cantilevers, recently developed tuning fork sensors ${ }^{52}$ could provide an alternative approach owing to their large stiffness. Considering the major biological relevance of hydrophilic/hydrophobic patches (often associated with the protein's activity ${ }^{48,49,51}$ ) coupled with the impressive advances on $3 \mathrm{D}$ imaging of hydration layers ${ }^{40,41,45,46}$ as well as more recent AFM techniques, ${ }^{52}$ it stands clear that this regime could offer major breakthroughs on the current spatial resolution limits.

All in all, our simulations provide a detailed lively picture of the indentation process on a single protein. The mechanisms unveiled put at evidence a wealth of challenges and opportunities for nanomechanical force spectroscopy which we hope that will stimulate new experiments to unlock the full potential of this technique.

\section{METHODS}

Atomic Level Models. The system here considered is composed by the following elements: (a) $20 \times 20 \mathrm{~nm}^{2}$ trilayer graphene slab with $A B A$ stacking; (b) a single immunoglobulin G (IgG) (Protein Data Bank (PDB) ID: $1 \mathrm{IGT}^{79}$ ) with zero net charge and protonation state such that $\mathrm{pH}=7.6$; (c) the tip, i.e., a diamond half-sphere with a radius of $2 \mathrm{~nm}$ and cleaved in such way that the (111) plane is parallel to the surface and it contains no dangling bonds; (d) the water molecules (192165 in total), where the system is inside a cubic box fully filled with TIP3P water molecules ${ }^{80}$ and whose size stretches 10 $\AA$ beyond (in each Cartesian direction) the furthest solute atom. The starting IgG adsorption configuration is taken from a previous work, ${ }^{37}$ which is in agreement with accompanying AFM experiments. The position of the atoms in the bottom graphene layer is restrained with a harmonic constant of $5 \mathrm{kcal} \cdot \mathrm{mol}^{-1} \AA^{-2}$. This setup mimics the typical configuration in many adsorption experiments where a graphene layer is supported on an inert, mechanically rigid substrate. The tip is positioned at nine different sites a-top the IgG (as described in the text) at a minimum initial distance of $1 \mathrm{~nm}$.

Force Fields. The protein and the associated oligosaccharide chains were modeled with the AMBER's $\mathrm{ff} 9 \mathrm{SB}^{81}$ and Glycam04 ${ }^{82}$ force fields, respectively. The choice of these force fields was motivated by a previous work, in which they reported ${ }^{50}$ that these force fields successfully sample the conformational space that an antibody explores in aqueous solution as well as its adsorption process to graphene. ${ }^{37}$ The carbon atoms of the three-layered graphene and the diamond tip were modeled using the OPLS aromatic (for the first) and aliphatic (for the second) carbon atom types present on AMBER's generalized AMBER force field. ${ }^{83}$ This force field properly describes graphene's mechanical and hydration properties, ${ }^{84}$ as well as its interaction with globular proteins. ${ }^{3,38,50,61,64}$ Additionally, wetting and tribological properties are also well-described with such a force field. ${ }^{85}$ The water molecules were modeled using the TIP3P water model, ${ }^{80}$ which is consistent with force fields used for the other components of the system.

Molecular Dynamics Simulation Details and Protocol. MD simulations were carried out using the AMBER14 software suite ${ }^{86}$ with NVIDIA GPU acceleration. ${ }^{87}$ Periodic boundary conditions and particle mesh Ewald (with standard defaults and a real-space cutoff of $1 \mathrm{~nm}$ ) were used to account for long-range electrostatic interactions. van der Waals interactions were truncated at the real-space cutoff, and Lorentz-Berthelot mixing rules were used to determine the interaction parameters between different atoms. A constant temperature of $300 \mathrm{~K}$ was ensured in all of the simulations by means of a Langevin thermostat. Before the indentation the pressure is stabilized for $10 \mathrm{~ns}$ in the NTP ensemble using Berendsen barostat to keep the pressure constant at $1 \mathrm{~atm}$. The SHAKE algorithm was used to constrain bonds containing hydrogen, thus allowing us to use an integration time step of $2 \mathrm{fs}$. Coordinates were saved every 1000 steps. In the steered MD simulations results employed to indent the protein, the conservative force $F(Z)$ was computed as $k_{\text {tip }}\left(Z-\left\langle Z_{\mathrm{p}}\right\rangle\right.$ ) (with $k_{\text {tip }}$ $=50 \mathrm{~N} / \mathrm{m})$, and its thermal average $\langle F(Z)\rangle$ approximated by a running average over $(100 \mathrm{ps})$, an interval adjusted to obtain a smooth dependence without distortions of the force. The resulting $\mathrm{d}\langle F(Z)\rangle / \mathrm{d} Z$ can be safely ${ }^{63,88}$ compared to the measured effective stiffness $k$ obtained in experiments. Our simulation protocol is composed by two stages. First we thermalize the system (equilibrate $T$ $=300 \mathrm{~K}$ and $P=1 \mathrm{~atm}$ ) during $10 \mathrm{~ns}$ while restraining the tipprotein distance to $1 \mathrm{~nm}$. Subsequently we indent, perpendicularly to the surface, in a NVT ensemble using constant velocity $(0.1 \mathrm{~m} / \mathrm{s})$ steered $\mathrm{MD}$-further details can be found in a previous work. ${ }^{85}$

\section{ASSOCIATED CONTENT}

\section{Supporting Information}

The Supporting Information is available free of charge at https://pubs.acs.org/doi/10.1021/acssensors.0c02241.

Figure S1, depicting the jump-to-contact observed in our simulations in the noncontact regime; Figure S2, comparing the flexibility measured at all indentation sites; and Figure S3, providing a schematic representation connecting hydrogen-bond network and protein stiffness (PDF)

\section{AUTHOR INFORMATION}

\section{Corresponding Authors}

J. G. Vilhena - Department of Physics, University of Basel, 4056 Basel, Switzerland; Departamento de Física Teórica de la Materia Condensada, Universidad Autónoma de Madrid, E-28049 Madrid, Spain; 이잉.org/0000-0001-83389119; Email: guilherme.vilhena@unibas.ch

Rubén Pérez - Departamento de Física Teórica de la Materia Condensada, Universidad Autónoma de Madrid, E-28049 Madrid, Spain; Condensed Matter Physics Center (IFIMAC), Universidad Autónoma de Madrid, E-28049 Madrid, Spain; ○ orcid.org/0000-0001-5896-541X; Email: ruben.perez@ uam.es

\section{Authors}

Maria Ortega - Departamento de Física Teórica de la Materia Condensada, Universidad Autónoma de Madrid, E-28049 Madrid, Spain 
Manuel R. Uhlig - Materials Science Factory, Instituto de Ciencia de Materiales de Madrid (ICMM), CSIC, 28049 Madrid, Spain

Ricardo Garcia - Materials Science Factory, Instituto de Ciencia de Materiales de Madrid (ICMM), CSIC, 28049 Madrid, Spain; orcid.org/0000-0002-7115-1928

Complete contact information is available at:

https://pubs.acs.org/10.1021/acssensors.0c02241

\section{Notes}

The authors declare no competing financial interest.

\section{ACKNOWLEDGMENTS}

J.G.V. acknowledges funding from a Marie Sklodowska-Curie Fellowship within the Horizon 2020 framework (Grant No. DLV-795286) and the Swiss National Science Foundation (Grant No. CRSK-2 190731/1). R.P. acknowledges support from the Spanish MINECO (Grant No. MAT2017-83273-R) and from the Ministerio de Ciencia e Innovación (MICINN) through the "María de Maeztu" Programme for Units of Excellence in R\&D (Grant No. CEX2018-000805-M). R.G. acknowledges funding from the MICINN (Grant No. PID2019-106801GB-I00) and Comunidad de Madrid Grant No. S2018/NMT-4443 (Tec4Bio-CM). We thankfully acknowledge the computer resources, technical expertise, and assistance provided by the Red Española de Supercomputación (RES) at the Minotauro and CTE-Power9 supercomputers (BSC, Barcelona). We thank Dr. Alejandro Martín-González for fruitful discussions.

\section{REFERENCES}

(1) Binnig, G.; Quate, C. F.; Gerber, C. Atomic force microscope. Phys. Rev. Lett. 1986, 56, 930-933.

(2) Dufrêne, Y. F.; Ando, T.; Garcia, R.; Alsteens, D.; MartinezMartin, D.; Engel, A.; Gerber, C.; Müller, D. J. Imaging modes of atomic force microscopy for application in molecular and cell biology. Nat. Nanotechnol. 2017, 12, 295-307.

(3) Gerber, C.; Lang, H. P. How the doors to the nanoworld were opened. Nat. Nanotechnol. 2006, 1, 3-5.

(4) Hörber, J. K.; Miles, M. J. Scanning Probe Evolution in Biology. Science 2003, 302, 1002-1005.

(5) Diao, Y.; Espinosa-Marzal, R. M. The role of water in fault lubrication. Nat. Commun. 2018, 9, 2309.

(6) Scholz, C. H. The Mechanics of Earthquakes and Faulting, 2nd ed.; Cambridge University Press, 2002; DOI: 10.1017/ CBO9780511818516.

(7) Krieg, M.; Fläschner, G.; Alsteens, D.; Gaub, B. M.; Roos, W. H.; Wuite, G. J.; Gaub, H. E.; Gerber, C.; Dufrêne, Y. F.; Müller, D. J. Atomic force microscopy-based mechanobiology. Nature Reviews Physics 2019, 1, 41-57.

(8) Rico, F.; Su, C.; Scheuring, S. Mechanical mapping of single membrane proteins at submolecular resolution. Nano Lett. 2011, 11, 3983-3986.

(9) Drake, B.; Prater, C. B.; Weisenhorn, A. L.; Gould, S. A.; Albrecht, T. R.; Quate, C. F.; Cannell, D. S.; Hansma, H. G.; Hansma, P. K. Imaging crystals, polymers, and processes in water with the atomic force microscope. Science 1989, 243, 1586-1589.

(10) Zhang, S.; Aslan, H.; Besenbacher, F.; Dong, M. Quantitative biomolecular imaging by dynamic nanomechanical mapping. Chem. Soc. Rev. 2014, 43, 7412-7429.

(11) Fukuma, T.; Kobayashi, K.; Matsushige, K.; Yamada, H. True atomic resolution in liquid by frequency-modulation atomic force microscopy. Appl. Phys. Lett. 2005, 87, 034101.

(12) Söngen, H.; Marutschke, C.; Spijker, P.; Holmgren, E.; Hermes, I.; Bechstein, R.; Klassen, S.; Tracey, J.; Foster, A. S.; Kühnle, A.
Chemical Identification at the Solid-Liquid Interface. Langmuir 2017, 33, 125-129.

(13) Reischl, B.; Watkins, M.; Foster, A. S. Free energy approaches for modeling atomic force microscopy in liquids. J. Chem. Theory Comput. 2013, 9, 600-608.

(14) Fukuma, T.; Reischl, B.; Kobayashi, N.; Spijker, P.; Canova, F. F.; Miyazawa, K.; Foster, A. S. Mechanism of atomic force microscopy imaging of three-dimensional hydration structures at a solid-liquid interface. Phys. Rev. B: Condens. Matter Mater. Phys. 2015, 92, 155412.

(15) Martinez-Martin, D.; Herruzo, E. T.; Dietz, C.; GomezHerrero, J.; Garcia, R. Noninvasive Protein Structural Flexibility Mapping by Bimodal Dynamic Force Microscopy. Phys. Rev. Lett. 2011, 106, 198101.

(16) Dong, M.; Husale, S.; Sahin, O. Determination of protein structural flexibility by microsecond force spectroscopy. Nat. Nanotechnol. 2009, 4, 514-517.

(17) Herrero-Galan, E.; Fuentes-Perez, M. E.; Carrasco, C.; Valpuesta, J. M.; Carrascosa, J. L.; Moreno-Herrero, F.; AriasGonzalez, J. R. Mechanical identities of RNA and DNA double helices unveiled at the single-molecule level. J. Am. Chem. Soc. 2013, 135, $122-131$

(18) Ando, T.; Uchihashi, T.; Fukuma, T. High-speed atomic force microscopy for nano-visualization of dynamic biomolecular processes. Prog. Surf. Sci. 2008, 83, 337-437.

(19) Preiner, J.; Kodera, N.; Tang, J.; Ebner, A.; Brameshuber, M.; Blaas, D.; Gelbmann, N.; Gruber, H. J.; Ando, T.; Hinterdorfer, P. IgGs are made for walking on bacterial and viral surfaces. Nat. Commun. 2014, 5, 4394.

(20) Martín-García, B.; Martín-González, A.; Carrasco, C.; Hernández-Arriaga, A. M.; Ruíz-Quero, R.; Díaz-Orejas, R.; AicartRamos, C.; Moreno-Herrero, F.; Oliva, M. A. The TubR-centromere complex adopts a double-ring segrosome structure in Type III partition systems. Nucleic Acids Res. 2018, 46, 5704-5716.

(21) Lyubchenko, Y. L.; Shlyakhtenko, L. S. AFM for analysis of structure and dynamics of DNA and protein-DNA complexes. Methods 2009, 47, 206-213.

(22) Garcia, R. Nanomechanical mapping of soft materials with the atomic force microscope: methods, theory and applications. Chem. Soc. Rev. 2020, 49, 5850-5884.

(23) Radmacher, M.; Cleveland, J. P.; Fritz, M.; Hansma, H. G.; Hansma, P. K. Mapping interaction forces with the atomic force microscope. Biophys. J. 1994, 66, 2159-2165.

(24) Radmacher, M.; Fritz, M.; Kacher, C. M.; Cleveland, J. P.; Hansma, P. K. Measuring the viscoelastic properties of human platelets with the atomic force microscope. Biophys. J. 1996, 70, 556567.

(25) Magonov, S. N.; Reneker, D. H. Characterization of polymer surfaces with atomic force microscopy. Annu. Rev. Mater. Sci. 1997, $27,175-222$.

(26) Mora, M.; Stannard, A.; Garcia-Manyes, S. The nanomechanics of individual proteins. Chem. Soc. Rev. 2020, 49, 6816-6832.

(27) Infante, E.; Stannard, A.; Board, S. J.; Rico-Lastres, P.; Rostkova, E.; Beedle, A. E.; Lezamiz, A.; Wang, Y. J.; Gulaidi Breen, S.; Panagaki, F.; et al. The mechanical stability of proteins regulates their translocation rate into the cell nucleus. Nat. Phys. 2019, 15, 973-981.

(28) Ido, S.; Kimiya, H.; Kobayashi, K.; Kominami, H.; Matsushige, K.; Yamada, H. Immunoactive two-dimensional self-assembly of monoclonal antibodies in aqueous solution revealed by atomic force microscopy. Nat. Mater. 2014, 13, 264-70.

(29) Pfreundschuh, M.; Martinez-Martin, D.; Mulvihill, E.; Wegmann, S.; Muller, D. J. Multiparametric high-resolution imaging of native proteins by force-distance curve-based AFM. Nat. Protoc. 2014, 9, 1113-1130.

(30) Müller, D. J.; Helenius, J.; Alsteens, D.; Dufrene, Y. F. Force probing surfaces of living cells to molecular resolution. Nat. Chem. Biol. 2009, 5, 383-390.

(31) Popa, I.; Berkovich, R.; Alegre-Cebollada, J.; Badilla, C. L.; Rivas-Pardo, J. A.; Taniguchi, Y.; Kawakami, M.; Fernandez, J. M. 
Nanomechanics of HaloTag tethers. J. Am. Chem. Soc. 2013, 135, 12762-12771.

(32) Valle-Orero, J.; Eckels, E. C.; Stirnemann, G.; Popa, I.; Berkovich, R.; Fernandez, J. M. The elastic free energy of a tandem modular protein under force. Biochem. Biophys. Res. Commun. 2015, 460, 434-438.

(33) Chowdhury, S. R.; Lu, H. P. Spontaneous Rupture and Entanglement of Human Neuronal Tau Protein Induced by Piconewton Compressive Force. ACS Chem. Neurosci. 2019, 10, 4061-4067.

(34) Chowdhury, S. R.; Cao, J.; He, Y.; Lu, H. P. Revealing Abrupt and Spontaneous Ruptures of Protein Native Structure under picoNewton Compressive Force Manipulation. ACS Nano 2018, 12, $2448-2454$.

(35) Perrino, A. P.; Garcia, R. How soft is a single protein? the stress-strain curve of antibody pentamers with $5 \mathrm{pN}$ and $50 \mathrm{pm}$ resolutions. Nanoscale 2016, 8, 9151-9158.

(36) Connolly, M. L. Analytical molecular surface calculation. J. Appl. Crystallogr. 1983, 16, 548-558.

(37) Vilhena, J. G.; Dumitru, A. C.; Herruzo, E. T.; MendietaMoreno, J. I.; Garcia, R.; Serena, P. A.; Pérez, R. Adsorption orientations and immunological recognition of antibodies on graphene. Nanoscale 2016, 8, 13463-13475.

(38) Ortega, M.; Vilhena, J. G.; Rubio-Pereda, P.; Serena, P. A.; Pérez, R. Assessing the Accuracy of Different Solvation Models to Describe Protein Adsorption. J. Chem. Theory Comput. 2019, 15, $2548-2560$.

(39) Kocun, M.; Labuda, A.; Meinhold, W.; Revenko, I.; Proksch, R. Fast, High Resolution, and Wide Modulus Range Nanomechanical Mapping with Bimodal Tapping Mode. ACS Nano 2017, 11, 1009710105.

(40) Umeda, K.; Zivanovic, L.; Kobayashi, K.; Ritala, J.; Kominami, H.; Spijker, P.; Foster, A. S.; Yamada, H. Atomic-resolution threedimensional hydration structures on a heterogeneously charged surface. Nat. Commun. 2017, 8, 2111.

(41) Uhlig, M. R.; Martin-Jimenez, D.; Garcia, R. Atomic-scale mapping of hydrophobic layers on graphene and few-layer MoS2 and WSe2 in water. Nat. Commun. 2019, 10, 2606.

(42) Hu, X.; Nanney, W.; Umeda, K.; Ye, T.; Martini, A. Combined Experimental and Simulation Study of Amplitude Modulation Atomic Force Microscopy Measurements of Self-Assembled Monolayers in Water. Langmuir 2018, 34, 9627-9633.

(43) Schlesinger, I.; Sivan, U. Three-Dimensional Characterization of Layers of Condensed Gas Molecules Forming Universally on Hydrophobic Surfaces. J. Am. Chem. Soc. 2018, 140, 10473-10481.

(44) Björneholm, O.; Hansen, M. H.; Hodgson, A.; Liu, L. M.; Limmer, D. T.; Michaelides, A.; Pedevilla, P.; Rossmeisl, J.; Shen, H.; Tocci, G.; et al. Water at Interfaces. Chem. Rev. 2016, 116, 76987726.

(45) Ricci, M.; Quinlan, R. A.; Voïtchovsky, K. Sub-nanometre mapping of the aquaporin-water interface using multifrequency atomic force microscopy. Soft Matter 2017, 13, 187-195.

(46) Fukuma, T.; Garcia, R. Atomic- and Molecular-Resolution Mapping of Solid-Liquid Interfaces by 3D Atomic Force Microscopy. ACS Nano 2018, 12, 11785-11797.

(47) Kuchuk, K.; Sivan, U. Hydration Structure of a Single DNA Molecule Revealed by Frequency-Modulation Atomic Force Microscopy. Nano Lett. 2018, 18, 2733-2737.

(48) Deng, Y.; Govers, C.; Teodorowicz, M.; Liobyte, I.; de Simone, I.; Hettinga, K.; Wichers, H. J. Hydrophobicity drives receptormediated uptake of heat-processed proteins by THP-1 macrophages and dendritic cells, but not cytokine responses. PLoS One 2020, 15, e0236212.

(49) Waku, T.; Nishigaki, S.; Kitagawa, Y.; Koeda, S.; Kawabata, K.; Kunugi, S.; Kobori, A.; Tanaka, N. Effect of the hydrophilichydrophobic balance of antigen-loaded peptide nanofibers on their cellular uptake, cellular toxicity, and immune stimulatory properties. Int. J. Mol. Sci. 2019, 20, 3781.
(50) Brandt, J. P.; Patapoff, T. W.; Aragon, S. R. Construction, MD simulation, and hydrodynamic validation of an all-atom model of a monoclonal IgG antibody. Biophys. J. 2010, 99, 905-913.

(51) Wang, J.-h.; Reinherz, E. L. The structural basis of $\alpha \beta$ T-lineage immune recognition: TCR docking topologies, mechanotransduction, and co-receptor function. Immunological Reviews 2012, 250, 102-119.

(52) Pürckhauer, K.; Weymouth, A. J.; Pfeffer, K.; Kullmann, L.; Mulvihill, E.; Krahn, M. P.; Müller, D. J.; Giessibl, F. J. Imaging in biologically-relevant environments with AFM using stiff qPlus sensors. Sci. Rep. 2018, 8, 9330.

(53) Ashby, M.; Hugh, S.; Cebon, D. Materials: Engineering, Science, Processing and Design, 4th ed.; Butterworth-Heinemann, 2019.

(54) Ackbarow, T.; Chen, X.; Keten, S.; Buehler, M. J. Hierarchies, multiple energy barriers, and robustness govern the fracture mechanics of $\alpha$-helical and $\beta$-sheet protein domains. Proc. Natl. Acad. Sci. U. S. A. 2007, 104, 16410-16415.

(55) Shahbazi, Z. Mechanical Model of Hydrogen Bonds in Protein Molecules. American Journal of Mechanical Engineering 2015, 3, 4754.

(56) Yu, H.; Siewny, M. G.; Edwards, D. T.; Sanders, A. W.; Perkins, T. T. Hidden dynamics in the unfolding of individual bacteriorhodopsin proteins. Science 2017, 355, 945-950.

(57) Garcia-Manyes, S.; Brujićacute, J.; Badilla, C. L.; Fernández, J. M. Force-clamp spectroscopy of single-protein monomers reveals the individual unfolding and folding pathways of i27 and ubiquitin. Biophys. J. 2007, 93, 2436-2446.

(58) Rief, M.; Gautel, M.; Oesterhelt, F.; Fernandez, J. M.; Gaub, H. E. Reversible unfolding of individual titin immunoglobulin domains by AFM. Science 1997, 276, 1109-1112.

(59) Cecconi, G.; Shank, E. A.; Bustamante, C.; Marqusee, S. Biochemistry: Direct observation of the three-state folding of a single protein molecule. Science 2005, 309, 2057-2060.

(60) Herruzo, E. T.; Perrino, A. P.; Garcia, R. Fast nanomechanical spectroscopy of soft matter. Nat. Commun. 2014, 5, 3126.

(61) Vilhena, J. G.; Rubio-Pereda, P.; Vellosillo, P.; Serena, P. A.; Pérez, R. Albumin (BSA) Adsorption over Graphene in Aqueous Environment: Influence of Orientation, Adsorption Protocol, and Solvent Treatment. Langmuir 2016, 32, 1742-1755.

(62) Ortega, M.; Vilhena, J. G.; Zotti, L. A.; Díez-Pérez, I.; Cuevas, J. C.; Pérez, R. Tuning structure and dynamics of blue copper azurin junctions via single amino-acid mutations. Biomolecules 2019, 9, 611.

(63) Pawlak, R.; Vilhena, J. G.; Hinaut, A.; Meier, T.; Glatzel, T.; Baratoff, A.; Gnecco, E.; Pérez, R.; Meyer, E. Conformations and cryo-force spectroscopy of spray-deposited single-strand DNA on gold. Nat. Commun. 2019, 10, 685.

(64) Rubio-Pereda, P.; Vilhena, J. G.; Takeuchi, N.; Serena, P. A.; Pérez, R. Albumin (BSA) adsorption onto graphite stepped surfaces. J. Chem. Phys. 2017, 146, 214704.

(65) Uhlig, M. R. Advanced force spectroscopy applications: Threedimensional AFM and single-molecule force spectroscopy. Ph.D. thesis, Universidad Autonoma de Madrid, Madrid, Spain, 2020.

(66) Bell, G. I. Models for the specific adhesion of cells to cells. Science 1978, 200, 618-627.

(67) Evans, E.; Ritchie, K. Dynamic strength of molecular adhesion bonds. Biophys. J. 1997, 72, 1541-1555.

(68) Bullerjahn, J. T.; Sturm, S.; Kroy, K. Theory of rapid force spectroscopy. Nat. Commun. 2014, 5, 4463.

(69) Marin-Gonzalez, A.; Vilhena, J. G.; Moreno-Herrero, F.; Perez, R. Sequence-dependent mechanical properties of double-stranded RNA. Nanoscale 2019, 11, 21471-21478.

(70) Marin-Gonzalez, A.; Vilhena, J. G.; Moreno-Herrero, F.; Perez, R. DNA Crookedness Regulates DNA Mechanical Properties at Short Length Scales. Phys. Rev. Lett. 2019, 122, 048102.

(71) Hansma, H. G.; Pietrasanta, L. I.; Golan, R.; Sitko, J. C.; Viani, M. B.; Paloczi, G. T.; Smith, B. L.; Thrower, D.; Hansma, P. K. Recent highlights from atomic force microscopy of DNA. J. Biomol. Struct. Dyn. 2000, 17, 271-275.

(72) Japaridze, A.; Muskhelishvili, G.; Benedetti, F.; Gavriilidou, A. F.; Zenobi, R.; De Los Rios, P.; Longo, G.; Dietler, G. Hyper- 
plectonemes: A Higher Order Compact and Dynamic DNA SelfOrganization. Nano Lett. 2017, 17, 1938-1948.

(73) Hansma, H. G.; Laney, D. E. DNA binding to mica correlates with cationic radius: Assay by atomic force microscopy. Biophys. J. 1996, 70, 1933-1939.

(74) Thomson, N. H.; Kasas, S.; Smith, B.; Hansma, H. G.; Hansma, P. K. Reversible binding of DNA to mica for AFM imaging. Langmuir 1996, 12, 5905-5906.

(75) Murugesapillai, D.; Bouaziz, S.; Maher, L. J.; Israeloff, N. E.; Cameron, C. E.; Williams, M. C. Accurate nanoscale flexibility measurement of DNA and DNA-protein complexes by atomic force microscopy in liquid. Nanoscale 2017, 9, 11327-11337.

(76) Ares, P.; Fuentes-Perez, M. E.; Herrero-Galán, E.; Valpuesta, J. M.; Gil, A.; Gomez-Herrero, J.; Moreno-Herrero, F. High resolution atomic force microscopy of double-stranded RNA. Nanoscale 2016, 8, $11818-11826$.

(77) Mou, J.; Czajkowsky, D. M.; Zhang, Y.; Shao, Z. Highresolution atomic-force microscopy of DNA: the pitch of the double helix. FEBS Lett. 1995, 371, 279-282.

(78) Kominami, H.; Kobayashi, K.; Yamada, H. Molecular-scale visualization and surface charge density measurement of Z-DNA in aqueous solution. Sci. Rep. 2019, 9, 6851.

(79) Harris, L. J.; Larson, S. B.; Hasel, K. W.; McPherson, A. Refined Structure of an Intact IgG2a Monoclonal Antibody. Biochemistry 1997, 36, 1581-1597.

(80) Jorgensen, W. L.; Chandrasekhar, J.; Madura, J. D.; Impey, R. W.; Klein, M. L. Comparison of simple potential functions for simulating liquid water. J. Chem. Phys. 1983, 79, 926-935.

(81) Lindorff-Larsen, K.; Piana, S.; Palmo, K.; Maragakis, P.; Klepeis, J. L.; Dror, R. O.; Shaw, D. E. Improved side-chain torsion potentials for the Amber ff99SB protein force field. Proteins: Struct., Funct., Genet. 2010, 78, 1950-1958.

(82) Kirschner, K. N.; Yongye, A. B.; Tschampel, S. M.; GonzálezOuteiriño, J.; Daniels, C. R.; Foley, B. L.; Woods, R. J. GLYCAM06: A generalizable biomolecular force field. Carbohydrate s. J. Comput. Chem. 2008, 29, 622-655.

(83) Wang, J.; Wolf, R. M.; Caldwell, J. W.; Kollman, P. A.; Case, D. A. Development and testing of a general amber force field. J. Comput. Chem. 2004, 25, 1157-1174.

(84) Tsai, J.-L.; Tu, J.-F. Characterizing mechanical properties of graphite using molecular dynamics simulation. Mater. Eng. 2010, 31, 194-199.

(85) Vilhena, J. G.; Pimentel, C.; Pedraz, P.; Luo, F.; Serena, P. A.; Pina, C. M.; Gnecco, E.; Pérez, R. Atomic-Scale Sliding Friction on Graphene in Water. ACS Nano 2016, 10, 4288-4293.

(86) Case, D. A.; Darden, T. A., III, T. E. C; Simmerling, C.; Wang, J.; Duke, R.; Luo, R.; Walker, R.; Zhang, W.; Merz, K.; et al. AMBER 14; University of California: San Francisco, 2014.

(87) Götz, A. W.; Williamson, M. J.; Xu, D.; Poole, D.; Le Grand, S.; Walker, R. C. Routine Microsecond Molecular Dynamics Simulations with AMBER on GPUs. 1. Generalized Born. J. Chem. Theory Comput. 2012, 8, 1542-1555.

(88) Pawlak, R.; Vilhena, J. G.; D’astolfo, P.; Liu, X.; Prampolini, G.; Meier, T.; Glatzel, T.; Lemkul, J. A.; Häner, R.; Decurtins, S.; et al. Sequential Bending and Twisting around C-C Single Bonds by Mechanical Lifting of a Pre-Adsorbed Polymer. Nano Lett. 2020, 20, 652-657. 